\title{
The Contrast Structures for a Class of Singularly Perturbed Systems with Heteroclinic Orbits
}

\author{
Han Xu and Yinlai Jin \\ School of Science, Linyi University, Linyi, Shandong 276005, China \\ Correspondence should be addressed to Yinlai Jin; jinyinlai@sina.com \\ Received 16 November 2015; Accepted 13 January 2016 \\ Academic Editor: Elvan Akın
}

Copyright (C) $2016 \mathrm{H}$. Xu and Y. Jin. This is an open access article distributed under the Creative Commons Attribution License, which permits unrestricted use, distribution, and reproduction in any medium, provided the original work is properly cited.

\begin{abstract}
Singularly perturbed problems are often used as the models of ecology and epidemiology. In this paper, a class of semilinear singularly perturbed systems with contrast structures are discussed. Firstly, we verify the existence of heteroclinic orbits connecting two equilibrium points about the associated systems for contrast structures in the corresponding phase space. Secondly, the asymptotic solutions of the contrast structures by the method of boundary layer functions and smooth connection are constructed. Finally, the uniform validity of the asymptotic expansion is defined and the existence of the smooth solutions is proved.
\end{abstract}

\section{Introduction}

Contrast structures in singularly perturbed problems can be classified as step-type contrast structures or spike-type contrast structures [1-3]. The existence of contrast structures is relevant to the existence of homoclinic orbits and heteroclinic orbits of their associated systems in [4-6]. Recently, contrast structures in singularly perturbed problems are attached great importance. $\mathrm{Ni}$ and Wang study four-dimensional contrast structures in singularly perturbed problems with fast variables in [7]. In [8], Wang considers a kind of step-type contrast structure for singularly perturbed problems with slow and fast variables and proves the existence of a heteroclinic orbit of its associated system in the corresponding phase space.

Since contrast structures can express the instantaneous transformation more accurately, we often use them in singularly perturbed problems as the models of the collision of cars and the transfer law of neurons. In [9], a kind of epidemical model with spike-type contrast structures is proposed. Chattoadhyay and Bairagi build an ecoepidemiological model in [10]:

$$
\begin{array}{ll}
\frac{d u}{d t}=d \Delta u+f_{1}(u, v), & (t, x) \in(0,+\infty) \times \Omega, \\
\frac{d v}{d t}=d \Delta v+f_{2}(u, v), & (t, x) \in(0,+\infty) \times \Omega,
\end{array}
$$

$$
\begin{aligned}
& \frac{d w}{d t}=d \Delta w+f_{3}(u, v), \quad(t, x) \in(0,+\infty) \times \Omega, \\
& \frac{\partial u}{\partial n}=\frac{\partial v}{\partial n}=\frac{\partial w}{\partial n}=0, \quad(t, x) \in(0,+\infty) \times \partial \Omega, \\
& u(x, 0) \geq 0, \quad v(x, 0) \geq 0, w(x, 0) \geq 0, x \in \Omega,
\end{aligned}
$$

where $d$ is a diffusion coefficient, $\Omega$ is a population habitat, $u$ is susceptible prey, $v$ is infected prey, $w$ is density of predators, and $n$ is a unit outer normal vector. By geometric methods and functional skills, Chattoadhyay and Bairagi study the existence of $u(x)$, which is a stable solution.

Considering the complexity of the ecoepidemiological model and the small parameter $d$, we propose the following semilinear singularly perturbed system with contrast structures:

$$
\begin{aligned}
\frac{d z}{d t} & =g(u, v, w, z, t), \\
\mu \frac{d u}{d t} & =f_{1}(u, v, w, z, t), \\
\mu \frac{d v}{d t} & =f_{2}(u, v, w, z, t), \\
\mu \frac{d w}{d t} & =f_{3}(u, v, w, z, t),
\end{aligned}
$$




$$
\begin{gathered}
u(0, \mu)=u^{0}, \\
v(0, \mu)=v^{0}, \\
w(1, \mu)=w^{1}, \\
z(0, \mu)=z^{0},
\end{gathered}
$$

where $0<\mu \ll 1,0 \leq t \leq 1$, the functions $f_{1}, f_{2}, f_{3}$, and $g$ are sufficiently smooth on the domain $\mathbf{G}=\{(u, v, w, z, t) \mid\|u\| \leq$ $\left.l_{1},\|v\| \leq l_{2},\|w\| \leq l_{3},\|z\| \leq l_{4}, 0 \leq t \leq 1\right\}$, and $l_{i}(i=1,2,3,4)$ are given positive real numbers. Assuming that $\mathbf{y}=(u, v, w)^{T}$ and $\mathbf{f}=\left(f_{1}, f_{2}, f_{3}\right)^{T}$, system (2) is equivalent to the following system:

$$
\begin{aligned}
\frac{d z}{d t} & =g(\mathbf{y}, z, t), \\
\mu \frac{d \mathbf{y}}{d t} & =\mathbf{f}(\mathbf{y}, z, t), \\
u(0, \mu) & =u^{0}, \\
v(0, \mu) & =v^{0}, \\
w(1, \mu) & =w^{1}, \\
z(0, \mu) & =z^{0} .
\end{aligned}
$$

The degenerate equations of (3) are

$$
\begin{aligned}
\mathbf{f}(\overline{\mathbf{y}}, \bar{z}, t) & =0, \\
\frac{d \bar{z}}{d t} & =g(\overline{\mathbf{y}}, \bar{z}, t) .
\end{aligned}
$$

In the following, we let

(A1) the degenerate equation $\mathbf{f}(\overline{\mathbf{y}}, \bar{z}, t)=0$ have two isolated smooth solutions $\overline{\mathbf{y}}=\alpha(\bar{z}, t)$ and $\overline{\mathbf{y}}=\beta(\bar{z}, t)$ on $D=\left\{(\bar{z}, t)|| \bar{z} \mid \leq l_{5}, 0 \leq t \leq 1\right\}$, where $\alpha=\left(\alpha_{1}, \alpha_{2}, \alpha_{3}\right)^{T}, \beta=\left(\beta_{1}, \beta_{2}, \beta_{3}\right)^{T}$, and $l_{5}$ is a given positive real number.

According to (A1), the initial value problem

$$
\frac{d \bar{z}^{(-)}}{d t}=g\left(\alpha\left(\bar{z}^{(-)}, t\right), \bar{z}^{(-)}, t\right), \quad \bar{z}^{(-)}(0)=z^{0},
$$

has the unique solution $\bar{z}^{(-)}(t)$ in $[0,1], 0<t_{0}<1$, and

$$
\frac{d \bar{z}^{(+)}}{d t}=g\left(\beta\left(\bar{z}^{(+)}, t\right), \bar{z}^{(+)}, t\right), \quad \bar{z}^{(+)}\left(t_{0}\right)=\bar{z}^{(-)}\left(t_{0}\right),
$$

has the unique solution $\bar{z}^{(+)}(t)$ in $[0,1]$. The associated system of (3) is

$$
\frac{d \widetilde{\mathbf{y}}}{d \tau}=\mathbf{f}(\widetilde{\mathbf{y}}, \bar{z}, \bar{t}), \quad \tau=\frac{\mu}{t} \geq 0
$$

where $\widetilde{\mathbf{y}}(\tau)=(\widetilde{u}(\tau), \widetilde{v}(\tau), \widetilde{w}(\tau))^{T}$ and $\bar{t}$ and $t$ are parameters. Obviously, system (7) exhibits two families of equilibrium points $M_{1}\left(\alpha\left(\bar{z}^{(-)}, \bar{t}\right), \bar{z}^{(-)}, \bar{t}\right)$ and $M_{2}\left(\beta\left(\bar{z}^{(+)}, \bar{t}\right), \bar{z}^{(+)}, \bar{t}\right)$. Assuming that $B_{l}(t)=\left.D_{\tilde{\mathbf{y}}} \mathbf{f}(\tilde{\mathbf{y}}, z, t)\right|_{M_{l}}, l=1,2$, we can classify sixteen cases (can be seen in $[11,12]$ ) on relations between $M_{1}$ and $M_{2}$ by the symbols of eigenvalues. There might exist interior layers satisfying one of the following cases:

(1) $M_{1}[-,-,+], M_{2}[-,-,+]$; (2) $M_{1}[-,+,+], M_{2}[-,+,+]$. We will discuss case (1); case (2) can be debated similarly.

(A2) Assume that $B_{l}(t)$ has three real-valued eigenvalues and satisfies the inequality $\lambda_{l 1}<\lambda_{l 2}<0<\lambda_{l 3}$.

By (A2), system (7) may exhibit a heteroclinic orbit connecting $M_{1}$ to $M_{2}$. To give the necessary conditions about the existence of a heteroclinic orbit, we introduce the following hypothesis:

(A3) The associated system (7) has two manifolds expressed by $\beta_{l}(\widetilde{\mathbf{y}}, \widetilde{z}, \bar{t})=C_{l}, l=1,2$.

The manifold crossing through $M_{1}$ is

$$
\Phi_{l}(\widetilde{\mathbf{y}}, \widetilde{z}, \bar{t})=\Phi_{l}^{(-)}\left(\alpha\left(\bar{z}^{(-)}, \bar{t}\right), \bar{z}^{(-)}, \bar{t}\right)
$$

The manifold crossing through $M_{2}$ is

$$
\Phi_{l}(\widetilde{\mathbf{y}}, \widetilde{z}, \bar{t})=\Phi_{l}^{(+)}\left(\beta\left(\bar{z}^{(+)}, \bar{t}\right), \bar{z}^{(+)}, \bar{t}\right)
$$

According to (8) and (9), the necessary conditions about the existence of heteroclinic orbits can be obtained by

$$
\Phi_{l}^{(-)}\left(\alpha\left(\bar{z}^{(-)}, \bar{t}\right), \bar{z}^{(-)}, \bar{t}\right)=\Phi_{l}^{(+)}\left(\beta\left(\bar{z}^{(+)}, \bar{t}\right), \bar{z}^{(+)}, \bar{t}\right) .
$$

By (A3), (8) and (9) can be expressed by

$$
\begin{aligned}
& \widetilde{v}^{(-)}(\tau)=\Psi_{2}^{(-)}\left(\tilde{u}^{(-)}, \bar{z}^{(-)}, \bar{t}\right), \\
& \widetilde{w}^{(-)}(\tau)=\Psi_{3}^{(-)}\left(\widetilde{u}^{(-)}, \bar{z}^{(-)}, \bar{t}\right), \\
& \widetilde{v}^{(+)}(\tau)=\Psi_{2}^{(+)}\left(\tilde{u}^{(+)}, \bar{z}^{(+)}, \bar{t}\right), \\
& \widetilde{w}^{(+)}(\tau)=\Psi_{3}^{(+)}\left(\tilde{u}^{(+)}, \bar{z}^{(+)}, \bar{t}\right) .
\end{aligned}
$$

Supposing that

$$
\begin{aligned}
& H_{h}(\bar{t})=\Psi_{h}^{(-)}\left(\tilde{u}^{(-)}, \bar{z}^{(-)}, \bar{t}\right)-\Psi_{h}^{(+)}\left(\tilde{u}^{(+)}, \bar{z}^{(+)}, \bar{t}\right) \\
& h=2,3 ;
\end{aligned}
$$

we can give the following hypothesis:

(A4) Assume that (12) has a solution of $\bar{t}=t_{0}$, and $\left.\left(\partial H_{2} / \partial \bar{t}\right)\right|_{\bar{t}=t_{0}},\left.\left(\partial H_{3} / \partial \bar{t}\right)\right|_{\bar{t}=t_{0}}$ are not simultaneously equal to zero.

In accordance with (A4), we can realize that $H_{h}\left(t_{0}\right)=0$, namely, there exists a heteroclinic orbit connecting $M_{1}$ and $M_{2}$.

Lemma 1. Under conditions (A1)-(A4), the associated system (7) has a heteroclinic orbit connecting $M_{1}$ and $M_{2}$, which is expressed by (10) and (12); therefore, system (3) has the solution with interior layer. 


\section{The Construction of Asymptotic Expansion}

In accordance with (A1)-(A4), system (3) has a step-like solution from $\alpha(t)$ to $\beta(t)$. Hence, we can suppose that

$$
\begin{gathered}
t^{*}=t_{0}+\mu t_{1}+\cdots+\mu^{k} t_{k}+\cdots, \\
\mathbf{x}\left(t^{*}, \mu\right)=\mathbf{x}_{0}^{*}+\mu \mathbf{x}_{1}^{*}+\cdots+\mu^{k} \mathbf{x}_{k}^{*}+\cdots,
\end{gathered}
$$

where $t^{*}$ is the transit point from $\alpha(t)$ to $\beta(t)$ and $t_{i}$ and $\mathbf{x}_{i}^{*}$, $i=1,2, \ldots$, are undetermined coefficients. The interior-layer solution of system (3) can be divided into two parts. The left problem is $\left(0 \leq t \leq t^{*}\right)$

$$
\begin{aligned}
\frac{d z^{(-)}}{d t} & =g\left(\mathbf{y}^{(-)}, z^{(-)}, t\right), \\
\mu \frac{d \mathbf{y}^{(-)}}{d t} & =\mathbf{f}\left(\mathbf{y}^{(-)}, z^{(-)}, t\right), \\
u^{(-)}(0, \mu) & =u^{0}, \\
v^{(-)}(0, \mu) & =v^{0}, \\
w^{(-)}\left(t^{*}, \mu\right) & =w^{*}, \\
z^{(-)}(0, \mu) & =z^{0} .
\end{aligned}
$$

The right problem is $\left(t^{*} \leq t \leq 1\right)$

$$
\begin{aligned}
\frac{d z^{(+)}}{d t} & =g\left(\mathbf{y}^{(+)}, z^{(+)}, t\right), \\
\mu \frac{d \mathbf{y}^{(+)}}{d t} & =\mathbf{f}\left(\mathbf{y}^{(+)}, z^{(+)}, t\right), \\
u^{(+)}\left(t^{*}, \mu\right) & =u^{*}, \\
v^{(+)}\left(t^{*}, \mu\right) & =v^{*}, \\
w^{(+)}(1, \mu) & =w^{1}, \\
z^{(+)}\left(t^{*}, \mu\right) & =z^{(-)}\left(t^{*}, \mu\right) .
\end{aligned}
$$

The step-like contrast structure of (3) can be regarded as the smooth connection at the point of $t^{*}$ by two solutions of (14) and (15). Let $\mathbf{x}=(u, v, w, z)^{T}$, and by the method of boundary layer functions [8-10], the asymptotic expansion of (14) can be constructed as follows:

$$
\begin{array}{r}
\mathbf{x}^{(-)}(t, \mu)=\sum_{k=0}^{\infty} \mu^{k}\left[\overline{\mathbf{x}}_{k}^{(-)}(t)+L_{k} \mathbf{x}\left(\tau_{0}\right)+Q_{k}^{(-)} \mathbf{x}(\tau)\right], \\
\tau_{0}=\frac{t}{\mu}, \tau=\frac{t-t^{*}}{\mu} .
\end{array}
$$

Also, the asymptotic expansion of (15) can be constructed as follows:

$$
\begin{array}{r}
\mathbf{x}^{(+)}(t, \mu)=\sum_{k=0}^{\infty} \mu^{k}\left[\overline{\mathbf{x}}_{k}^{(+)}(t)+Q_{k}^{(+)} \mathbf{x}(\tau)+R_{k} \mathbf{x}\left(\tau_{1}\right)\right], \\
\tau_{1}=\frac{t-1}{\mu},
\end{array}
$$

where $\overline{\mathbf{x}}_{k}^{( \pm)}(t)$ are the coefficients of regular series, $L_{k} \mathbf{x}\left(\tau_{0}\right)$ are the coefficients of left boundary layer series, $R_{k} \mathbf{x}\left(\tau_{1}\right)$ are the coefficients of right boundary layer series, and $Q_{k}^{( \pm)} \mathbf{x}(\tau)$ are the coefficients of interior-layer series.

Putting (16) and (17) into (14) and (15), and separating equations by scales $t, \tau_{0}, \tau$, and $\tau_{1}$, then $\overline{\mathbf{x}}_{0}^{( \pm)}(t)$ satisfies

$$
\begin{aligned}
\mathbf{f}\left(\overline{\mathbf{y}}_{0}^{(-)}, \bar{z}_{0}^{(-)}, t\right) & =0, \\
\left(\bar{z}_{0}^{(-)}\right)^{\prime} & =g\left(\overline{\mathbf{y}}_{0}^{(-)}, \bar{z}_{0}^{(-)}, t\right), \\
\bar{z}_{0}^{(-)}(0) & =z^{0}, \\
\mathbf{f}\left(\overline{\mathbf{y}}_{0}^{(+)}, \bar{z}_{0}^{(+)}, t\right) & =0, \\
\left(\bar{z}_{0}^{(+)}\right)^{\prime} & =g\left(\overline{\mathbf{y}}_{0}^{(+)}, \bar{z}_{0}^{(+)}, t\right), \\
\bar{z}_{0}^{(+)}\left(t_{0}\right) & =\bar{z}_{0}^{(-)}\left(t_{0}\right) .
\end{aligned}
$$

By (A2), we can obtain that $\bar{z}_{0}^{(-)}=\bar{z}^{(-)}(t), \overline{\mathbf{y}}_{0}^{(-)}=$ $\alpha\left(\bar{z}^{(-)}(t), t\right)=\alpha\left(t, t_{0}\right), \bar{z}_{0}^{(+)}=\bar{z}^{(+)}(t)$, and $\overline{\mathbf{y}}_{0}^{(+)}=\beta\left(\bar{z}^{(+)}(t), t\right)=$ $\beta\left(t, t_{0}\right)$, where $z^{( \pm)}(t)$ are functions about $t$ and $t_{0}$ and $t_{0}$ is an undetermined constant.

For $Q_{0}^{(-)}(\tau)$, we have

$$
\begin{aligned}
& \frac{d Q_{0}^{(-)} z(\tau)}{d \tau}=0, \\
& \frac{d Q_{0}^{(-)} \mathbf{y}(\tau)}{d \tau} \\
& \quad=\mathbf{f}\left(\alpha\left(t_{0}\right)+Q_{0}^{(-)} \mathbf{y}(\tau), \bar{z}_{0}^{(-)}+Q_{0}^{(-)} z(\tau), t_{0}\right), \\
& \alpha_{1}\left(t_{0}\right)+Q_{0}^{(-)} u(0)=u^{*}, \\
& Q_{0}^{(-)} \mathbf{x}(-\infty)=0 .
\end{aligned}
$$

By (20), we can solve $Q_{0}^{(-)} z(\tau) \equiv 0$. Assuming that $\widetilde{\mathbf{y}}^{l}=\alpha\left(t_{0}\right)+$ $Q_{0}^{(-)} \mathbf{y}(\tau),(20)$ can be rewritten as

$$
\begin{aligned}
\frac{d \widetilde{\mathbf{y}}^{l}}{d \tau} & =\mathbf{f}\left(\widetilde{\mathbf{y}}^{l}, \bar{z}_{0}^{(-)}\left(t_{0}\right), t_{0}\right), \\
\widetilde{u}^{l}(0) & =u^{*}, \\
\widetilde{\mathbf{y}}^{l}(-\infty) & =\alpha\left(t_{0}\right) .
\end{aligned}
$$

For $Q_{0}^{(+)}(\tau)$, we have

$$
\begin{aligned}
& \frac{d Q_{0}^{(+)} z(\tau)}{d \tau}=0, \\
& \frac{d Q_{0}^{(+)} \mathbf{y}(\tau)}{d \tau} \\
& \quad=\mathbf{f}\left(\beta\left(t_{0}\right)+Q_{0}^{(+)} \mathbf{y}(\tau), \bar{z}_{0}^{(+)}+Q_{0}^{(+)} z(\tau), t_{0}\right), \\
& \beta_{1}\left(t_{0}\right)+Q_{0}^{(+)} u(0)=u^{*}, \\
& Q_{0}^{(+)} \mathbf{x}(+\infty)=0 .
\end{aligned}
$$


By (22), we can solve $Q_{0}^{(+)} z(\tau) \equiv 0$. Supposing that $\widetilde{\mathbf{y}}^{r}=$ $\beta\left(t_{0}\right)+Q_{0}^{(+)} \mathbf{y}(\tau)$, (22) can be rewritten as

$$
\begin{aligned}
\frac{d \widetilde{\mathbf{y}}^{r}}{d \tau} & =\mathbf{f}\left(\widetilde{\mathbf{y}}^{r}, \bar{z}_{0}^{(+)}\left(t_{0}\right), t_{0}\right), \\
\widetilde{u}^{r}(0) & =u^{*}, \\
\widetilde{\mathbf{y}}^{r}(+\infty) & =\beta\left(t_{0}\right) .
\end{aligned}
$$

Obviously, systems (21) and (23) coincide with the associated system (7), so we can consider their combined system

$$
\begin{aligned}
\frac{d \widetilde{\mathbf{y}}}{d \tau} & =\mathbf{f}\left(\widetilde{\mathbf{y}}, \bar{z}_{0}\left(t_{0}\right), t_{0}\right), \\
\widetilde{u}(0) & =u^{*}, \\
\widetilde{\mathbf{y}}(+\infty) & =\beta\left(t_{0}\right), \\
\widetilde{\mathbf{y}}(-\infty) & =\alpha\left(t_{0}\right) .
\end{aligned}
$$

By (A1), (A2), and (A3), system (24) has a solution, which is a heteroclinic orbit connecting $M_{1}\left(\alpha\left(t_{0}\right), \bar{z}_{0}^{(-)}\left(t_{0}\right), t_{0}\right)$ with $M_{2}\left(\beta\left(t_{0}\right), \bar{z}_{0}^{(+)}\left(t_{0}\right), t_{0}\right)$. On the basis of (A4) and (10), the value of $t_{0}$ can be confirmed, so $Q_{0}^{( \pm)} \mathbf{y}(\tau)$ is determined completely.

For $L_{0} \mathbf{x}\left(\tau_{0}\right)$, we have

$$
\begin{aligned}
& \frac{d L_{0} z\left(\tau_{0}\right)}{d \tau_{0}}=0, \\
& \frac{d L_{0} \mathbf{y}\left(\tau_{0}\right)}{d \tau_{0}} \\
& \quad=\mathbf{f}\left(\alpha(0)+L_{0} \mathbf{y}\left(\tau_{0}\right), \bar{z}_{0}^{(-)}(0)+L_{0} z\left(\tau_{0}\right), 0\right), \\
& L_{0} u(0)=u^{*}-\alpha_{1}(0), \\
& L_{0} v(0)=v^{0}-\alpha_{2}(0), \\
& L_{0} \mathbf{x}(+\infty)=0 .
\end{aligned}
$$

By (25), we can solve $L_{0} z\left(\tau_{0}\right) \equiv 0$. Assuming that $\widetilde{\widetilde{\mathbf{y}}}^{l}=\alpha(0)+$ $L_{0} \mathbf{y}\left(\tau_{0}\right),(25)$ can be rewritten as

$$
\begin{aligned}
& \frac{d \widetilde{\widetilde{\mathbf{y}}}^{l}}{d \tau_{0}}=\mathbf{f}\left(\widetilde{\widetilde{\mathbf{y}}}^{l}, \bar{z}_{0}^{(-)}(0), 0\right), \\
\tilde{\widetilde{u}}^{l}(0) & =u^{0}, \\
\widetilde{\widetilde{v}}^{l}(0) & =v^{0}, \\
\widetilde{\widetilde{\mathbf{y}}}^{l}(+\infty) & =\alpha(0) .
\end{aligned}
$$

For $R_{0} \mathbf{x}\left(\tau_{1}\right)$, we have

$$
\begin{aligned}
& \frac{d R_{0} z\left(\tau_{1}\right)}{d \tau_{1}}=0, \\
& \frac{d R_{0} \mathbf{y}\left(\tau_{1}\right)}{d \tau_{1}} \\
& \quad=\mathbf{f}\left(\beta(1)+R_{0} \mathbf{y}\left(\tau_{1}\right), \bar{z}_{0}^{(+)}(1)+R_{0} z\left(\tau_{1}\right), 1\right), \\
& R_{0} w(0)=w^{1}-\beta_{3}(1), \\
& R_{0} \mathbf{x}(-\infty)=0 .
\end{aligned}
$$

According to (27), we can solve $R_{0} z\left(\tau_{1}\right) \equiv 0$. Supposing that $\tilde{\widetilde{\mathbf{y}}}^{r}=\beta(1)+R_{0} \mathbf{y}\left(\tau_{1}\right),(27)$ can be rewritten as

$$
\begin{aligned}
& \frac{d \widetilde{\mathbf{y}}^{r}}{d \tau_{1}}=\mathbf{f}\left(\widetilde{\widetilde{\mathbf{y}}}^{r}, \bar{z}_{0}^{(+)}(1), 1\right), \\
& \widetilde{\widetilde{w}}^{r}(0)=w^{1}, \\
& \widetilde{\widetilde{\mathbf{y}}}^{r}(-\infty)=\beta(1) .
\end{aligned}
$$

Equations (26) and (28) coincide with the associated system (7). By (A1), systems (26) and (28) have the equilibrium points $\widetilde{M}_{1}\left(\varphi(0), \bar{z}_{0}^{(-)}(0), 0\right)$ and $\widetilde{M}_{2}\left(\psi(1), \bar{z}_{0}^{(+)}(1), 1\right)$, respectively. We will give the following hypothesis to obtain the solution of systems (26) and (28):

(A5) The initial values $\widetilde{\widetilde{u}}^{l}(0)=u^{0}$ and $\widetilde{\widetilde{v}}^{l}(0)=v^{0}$ are intersected with the one-dimensional stable manifold $W^{s}\left(\widetilde{M}_{1}(0)\right)$ near the equilibrium point $\widetilde{M}_{1}$, and the initial value $\widetilde{\widetilde{w}}^{r}(0)=w^{1}$ is intersected with the onedimensional unstable manifold $W^{u}\left(\widetilde{M}_{2}(1)\right)$.

By (A1)-(A4) and (20)-(24), $Q_{0}^{( \pm)} \mathbf{x}(\tau)$ is solved, which decays exponentially as $\tau \rightarrow \pm \infty$. Then, by (A5) and (25)(28), we can solve $L_{0} \mathbf{x}\left(\tau_{0}\right)$, which decays exponentially as $\tau \rightarrow+\infty$, and $R_{0} z\left(\tau_{1}\right)$, which decays exponentially as $\tau \rightarrow$ $-\infty$. So, the following conclusion is obtained.

Lemma 2. Under conditions (A1)-(A5) and (20)-(28), there exist the interior-layer functions $Q_{0}^{( \pm)} \mathbf{x}(\tau)$ and the boundary layer functions $L_{0} \mathbf{x}\left(\tau_{0}\right), R_{0} \mathbf{x}\left(\tau_{1}\right)$, which satisfy the following inequality:

$$
\begin{gathered}
\left\|Q_{0}^{(-)} \mathbf{x}(\tau)\right\| \leq C_{0} e^{k_{0} \tau}, \\
\left\|Q_{0}^{(+)} \mathbf{x}(\tau)\right\| \leq C_{1} e^{-k_{1} \tau}, \\
\left\|L_{0} \mathbf{x}\left(\tau_{0}\right)\right\| \leq C_{2} e^{-k_{2} \tau_{0}}, \\
\left\|R_{0} \mathbf{x}\left(\tau_{1}\right)\right\| \leq C_{3} e^{k_{3} \tau_{1}},
\end{gathered}
$$

where $C_{l}$ and $k_{l}(l=0,1,2,3)$ are all positive constants.

Now, the coefficients of zero-order terms for (16) and (17) are completely determined. To determine functions $\overline{\mathbf{y}}_{i}^{( \pm)}(t)$ and $\bar{z}_{i}^{( \pm)}(t)$, we need the following hypothesis: 
(A6) The determinant of $\mathbf{f}_{\mathbf{y}}\left(\overline{\mathbf{y}}_{0}^{( \pm)}(t), \bar{z}_{0}^{( \pm)}, t\right)$ is not equal to zero all the time.

For $\overline{\mathbf{x}}_{i}^{( \pm)}(t)$, we can obtain

$$
\begin{aligned}
& \left(\overline{\mathbf{y}}_{i-1}^{( \pm)}(t)\right)^{\prime}=\mathbf{f}_{y} \overline{\mathbf{y}}_{i}^{( \pm)}(t)+\mathbf{f}_{z} \bar{z}_{i}^{( \pm)}(t)+h_{i}(t), \\
& \left(\bar{z}_{i}^{( \pm)}(t)\right)^{\prime}=g_{y} \overline{\mathbf{y}}_{i}^{( \pm)}(t)+g_{z} \bar{z}_{i}^{( \pm)}(t)+g_{i}(t), \\
& \bar{z}_{i}^{(-)}(0)=-\Pi_{i} z(0), \\
& \bar{z}_{i}^{(-)}\left(t_{0}\right)+Q_{i}^{(-)} z(0)+\sum_{k=1}^{i}\left[\bar{z}_{i-k}^{(-)}\left(t_{0}\right)\right]^{(k)} t_{k} \\
& =\bar{z}_{i}^{(+)}\left(t_{0}\right)+Q_{i}^{(+)} z(0)+\sum_{k=1}^{i}\left[\bar{z}_{i-k}^{(+)}\left(t_{0}\right)\right]^{(k)} t_{k},
\end{aligned}
$$

where functions $\mathbf{f}_{y}, \mathbf{f}_{z}, g_{y}$, and $g_{z}$ take value at $\left(\overline{\mathbf{y}}_{0}^{( \pm)}(t), \bar{z}_{0}^{( \pm)}, t\right)$, while $h_{i}(t)$ and $g_{i}(t)$ are known functions about $\overline{\mathbf{y}}_{m}(t)$, $\bar{z}_{m}(t)(m=0,1,2, \ldots, i-1)$. On the basis of (A6) and the first equation of (30), we can ascertain

$$
\overline{\mathbf{y}}_{i}^{( \pm)}(t)=\mathbf{f}_{y}^{-1}\left[\left(\overline{\mathbf{y}}_{i-1}^{( \pm)}(t)\right)^{\prime}-\mathbf{f}_{z} \bar{z}_{i}^{( \pm)}(t)-h_{i}(t)\right] .
$$

Inserting $\overline{\mathbf{y}}_{i}^{( \pm)}(t)$ into the second equations of $(30), \bar{z}_{i}^{( \pm)}(t, c)$ is solved, where $c$ is an undetermined coefficient.

For $L_{i} \mathbf{x}\left(\tau_{0}\right) \quad(i=1,2, \ldots)$, we can obtain

$$
\begin{aligned}
\frac{d L_{i} \mathbf{y}}{d \tau_{0}} & =\widetilde{\mathbf{f}}_{y} L_{i} \mathbf{y}+\widetilde{\mathbf{f}}_{z} L_{i} z+G_{i}\left(\tau_{0}\right), \\
\frac{d L_{i} z}{d \tau_{0}} & =\widetilde{g}_{y} L_{i-1} \mathbf{y}+\widetilde{g}_{z} L_{i-1} z+M_{i-1}\left(\tau_{0}\right) . \\
L_{i} u(0) & =-\bar{u}_{i}(0), \\
L_{i} \mathbf{x}(+\infty) & =0,
\end{aligned}
$$

where $\widetilde{\mathbf{f}}_{y}, \widetilde{\mathbf{f}}_{z}, \widetilde{g}_{y}$, and $\widetilde{g}_{z}$ take value at $\left(\alpha(0)+L_{0} \mathbf{y}\left(\tau_{0}\right), \bar{z}_{0}(0)+\right.$ $\left.L_{0} z\left(\tau_{0}\right), 0\right), G_{i}(\tau)$ is a known vector function about $\overline{\mathbf{x}}_{i-1}(t)$ and $L_{i-1} \mathbf{x}\left(\tau_{0}\right)$, and $M_{i-1}\left(\tau_{0}\right)$ is a known vector function about $\overline{\mathbf{x}}_{i-2}(t)$ and $L_{i-2} \mathbf{x}\left(\tau_{0}\right)$. By the second equation of (32), we can solve $L_{i} z\left(\tau_{0}\right)=\int_{+\infty}^{\tau_{0}} L_{i-1} g(s) d s$, where $L_{i-1} g\left(\tau_{0}\right)=\widetilde{g}_{y} L_{i-1} \mathbf{y}+$ $\tilde{g}_{z} L_{i-1} z+M_{i-1}\left(\tau_{0}\right)$, so $L_{i} z(0)=\int_{+\infty}^{0} L_{i-1} g(s) d s$. By (30), we can know

$$
\bar{z}_{i}^{(-)}(t)=-L_{i} z(0)=\int_{0}^{+\infty} L_{i-1} g(s) d s
$$

By the above condition and $(28), \overline{\mathbf{x}}_{i}^{( \pm)}(t)$ is confirmed; similarly, we can solve (32).
For $Q_{i}^{( \pm)} \mathbf{x}(\tau)(i=1,2, \ldots)$, we can obtain

$$
\begin{aligned}
\frac{d Q_{i}^{( \pm)} \mathbf{y}}{d \tau} & =\widetilde{\mathbf{f}}_{y}^{( \pm)} Q_{i}^{( \pm)} \mathbf{y}+\widetilde{\mathbf{f}}_{z}^{( \pm)} Q_{i}^{( \pm)} z+H_{i}^{( \pm)}(\tau), \\
\frac{d Q_{i}^{( \pm)} z}{d \tau} & =\widetilde{g}_{y}^{( \pm)} Q_{i-1} \mathbf{y}+\widetilde{g}_{z}^{( \pm)} Q_{i-1} z+N_{i-1}^{( \pm)}(\tau), \\
Q_{i}^{( \pm)} u(0) & =u_{i}^{*}-\sum_{k=0}^{i}\left[\bar{u}_{i-k}^{( \pm)}\left(t_{0}\right)\right]^{(k)} t_{k}, \\
Q_{i}^{(-)} \mathbf{x}(-\infty) & =Q_{i}^{(+)} \mathbf{x}(+\infty)=0,
\end{aligned}
$$

where $\widetilde{\mathbf{f}}_{y}^{(-)}, \widetilde{\mathbf{f}}_{z}^{(-)}$, and $\widetilde{g}_{y}^{( \pm)}$take value at $\left(\alpha\left(t_{0}\right)+Q_{0}^{(-)} \mathbf{y}(\tau)\right.$, $\left.\bar{z}_{0}^{(-)}\left(t_{0}\right)+Q_{0}^{(-)} z(\tau), t_{0}\right)$ and $\widetilde{\mathbf{f}}_{y}^{(+)}, \widetilde{\mathbf{f}}_{z}^{(+)}$, and $\widetilde{g}_{z}$ take value at $\left(\beta\left(t_{0}\right)+Q_{0}^{(+)} \mathbf{y}(\tau), \bar{z}_{0}^{(+)}\left(t_{0}\right)+Q_{0}^{(+)} z(\tau), t_{0}\right) \cdot H_{i}^{( \pm)}(\tau)$ is a known vector function about $\overline{\mathbf{x}}_{i-n}^{( \pm)}(t), Q_{i-n}^{( \pm)} \mathbf{x}(\tau)$, and $t_{i-n}(0 \leq n \leq$ $i-1)$. By the condition $Q_{i}^{(-)} z(-\infty)=0$ and the second equation of (34), we can solve $Q_{i}^{(-)} z(\tau)=\int_{-\infty}^{\tau} Q_{i-1}^{(-)} g(s) d s$, where $Q_{i-1}^{( \pm)} g(\tau)=\tilde{g}_{y}^{( \pm)} Q_{i-1} \mathbf{y}+\tilde{g}_{z}^{( \pm)} Q_{i-1} z+N_{i-1}^{( \pm)}(\tau)$. We can know that

$$
Q_{i}^{(-)} z(0)=\int_{-\infty}^{0} Q_{i-1}^{(-)} g(s) d s .
$$

By (35) and the first equation of (34), $Q_{i}^{(-)} \mathbf{x}(\tau)$ is solved, which decays exponentially as $\tau \rightarrow-\infty$. Then, we can solve $Q_{i}^{(+)} \mathbf{x}(\tau)$ which decays exponentially as $\tau \rightarrow+\infty$. Inserting (35) into the second condition of (30), $t_{i}$ is solved.

The boundary value function $R_{i} \mathbf{x}\left(\tau_{1}\right)$ satisfies the following equations:

$$
\begin{aligned}
\frac{d R_{i} \mathbf{y}}{d \tau_{1}} & =\widetilde{\mathbf{f}}_{y} R_{i} \mathbf{y}+\widetilde{\mathbf{f}}_{z} R_{i} z+G_{i}\left(\tau_{1}\right), \\
\frac{d R_{i} z}{d \tau_{1}} & =\widetilde{g}_{y} R_{i-1} \mathbf{y}+\widetilde{g}_{z} R_{i-1} z+M_{i-1}\left(\tau_{1}\right) . \\
R_{i} w(0) & =w^{1}-\bar{w}_{i}(1), \\
R_{i} \mathbf{x}(-\infty) & =0 .
\end{aligned}
$$

The solution of (36) is similar to the solution of (32). From (36), we can verify that there exists the right boundary value function $R_{i} \mathbf{x}\left(\tau_{1}\right)$, which decays exponentially as $\tau \rightarrow-\infty$, and the following conclusion is obtained.

Lemma 3. Systems (32)-(36) have the solutions $L_{i} \mathbf{x}\left(\tau_{0}\right)$, $Q_{i}^{( \pm)} \mathbf{x}(\tau)$, and $R_{i} \mathbf{x}\left(\tau_{1}\right)$, respectively, satisfying the following inequality:

$$
\begin{aligned}
\left\|L_{i} \mathbf{x}\left(\tau_{0}\right)\right\| & \leq \bar{C}_{0} e^{-\bar{k}_{0} \tau_{0}}, \\
\left\|Q_{i}^{(-)} \mathbf{x}(\tau)\right\| & \leq \bar{C}_{1} e^{\bar{k}_{1} \tau}, \\
\left\|Q_{i}^{(+)} \mathbf{x}(\tau)\right\| & \leq \bar{C}_{2} e^{-\bar{k}_{2} \tau}, \\
\left\|R_{i} \mathbf{x}\left(\tau_{1}\right)\right\| & \leq \bar{C}_{3} e^{\bar{k}_{3} \tau_{1}},
\end{aligned}
$$

where $\bar{C}_{l}$ and $\bar{k}_{l}(l=0,1,2,3)$ are all positive constants. 


\section{The Existence of Asymptotic Expansion}

There are many methods to prove the existence of the steplike contrast structure for system (3) and we will prove the existence with implicit functions theorems [11]. The solutions of left and right problems can be expressed by (15) and (16) and we will prove that (15) and (16) are connected smoothly at the point of $t^{*}$, which is on the neighborhood of $t_{0}$. At the point of $t^{*},(15)$ and (16) can be expressed by

$$
\begin{aligned}
& \mathbf{x}^{(-)}\left(t^{*}, \mu\right)=\overline{\mathbf{x}}_{0}^{(-)}\left(t^{*}\right)+Q_{0}^{(-)} \mathbf{x}(0)+O(\mu), \\
& \mathbf{x}^{(+)}\left(t^{*}, \mu\right)=\overline{\mathbf{x}}_{0}^{(+)}\left(t^{*}\right)+Q_{0}^{(+)} \mathbf{x}(0)+O(\mu) .
\end{aligned}
$$

As $L_{0} \mathbf{x}\left(\tau_{0}\right)$ and $R_{0} \mathbf{x}\left(\tau_{1}\right)$ decay exponentially as $t=t^{*}$, they can be neglected. Because the first two components of the solution for the left and right problems are equal correspondingly at the point of $t^{*}$, we can solve $t^{*}$ by the third component $w^{( \pm)}$. Supposing that $U\left(t^{*}, \mu\right)=u^{(-)}\left(t^{*}, \mu\right)-$ $u^{(+)}\left(t^{*}, \mu\right)$, we have

$$
\begin{aligned}
U\left(t^{*}, \mu\right)= & {\left[\alpha_{3}\left(t^{*}\right)+Q_{0}^{(-)} w(0)\right] } \\
& -\left[\beta_{3}\left(t^{*}\right)+Q_{0}^{(+)} w(0)\right]+O(\mu) \\
= & H_{3}\left(t^{*}\right)+O(\mu) .
\end{aligned}
$$

According to (A4), $\left.\left(\partial H_{2} / \partial \bar{t}\right)\right|_{\bar{t}=t_{0}}$ and $\left.\left(\partial H_{3} / \partial \bar{t}\right)\right|_{\bar{t}=t_{0}}$ are not simultaneously equal to zero. If $\left.\left(\partial \mathrm{H}_{2} / \partial \bar{t}\right)\right|_{\bar{t}=t_{0}}=0$, we can ascertain that $\left.\left(\partial H_{3} / \partial \bar{t}\right)\right|_{\bar{t}=t_{0}} \neq 0$. On the basis of the implicit functions theorem, there exists $t^{*}(\mu)=t_{0}+O(\mu)$ causing $U\left(t^{*}, \mu\right)=0$. So there exists a step-like contrast structure at the point of $t^{*}$. If $\left.\left(\partial H_{2} / \partial \bar{t}\right)\right|_{\bar{t}=t_{0}} \neq 0,\left.\left(\partial H_{3} / \partial \bar{t}\right)\right|_{\bar{t}=t_{0}}=0$ and $t^{*}$ can be confirmed by the second component $v$. By the above discussion, the following theorem is obtained.

Theorem 4. By (A1)-(A6) and Lemmas 1-3, there exists the step-like solution of (3) as follows:

$$
\begin{aligned}
& \mathbf{x}(t, \mu) \\
& = \begin{cases}\overline{\mathbf{x}}_{0}^{(-)}(t)+L_{0} \mathbf{x}\left(\tau_{0}\right)+Q_{0}^{(-)} \mathbf{x}(\tau)+O(\mu), & 0 \leq t \leq t^{*}, \\
\overline{\mathbf{x}}_{0}^{(+)}(t)+Q_{0}^{(+)} \mathbf{x}(\tau)+R_{0} \mathbf{x}\left(\tau_{1}\right)+O(\mu), & t^{*} \leq t \leq 1 .\end{cases}
\end{aligned}
$$

\section{Example}

The equations are given by

$$
\begin{aligned}
\mu \frac{d u}{d t} & =v, \\
\mu \frac{d v}{d t} & =\left(u-t+\frac{1}{2}\right)\left(u^{2}-\frac{9}{4}\right), \\
\mu \frac{d w}{d t} & =\frac{3}{\sqrt{2}}(u-w), \\
\frac{d z}{d t} & =u+z,
\end{aligned}
$$

satisfying the boundary value conditions as follows:

$$
\begin{gathered}
u(0, \mu)=u^{0}, \\
v(0, \mu)=v^{0}, \\
w(1, \mu)=w^{1}, \\
z(0, \mu)=z^{0} .
\end{gathered}
$$

Assuming that $\mu=0$, we can solve three groups of isolated solutions:

$$
\begin{aligned}
& \overline{\mathbf{y}}=\alpha(t)=\left(-\frac{3}{2}, 0,-\frac{3}{2}\right)^{T}, \\
& \overline{\mathbf{y}}=\chi(t)=\left(t-\frac{1}{2}, 0, t-\frac{1}{2}\right)^{T}, \\
& \overline{\mathbf{y}}=\beta(t)=\left(\frac{3}{2}, 0, \frac{3}{2}\right)^{T} .
\end{aligned}
$$

According to (43), the solution of $d z / d t=u+z$ exists. Considering the associated system,

$$
\begin{aligned}
& \frac{d \tilde{u}}{d \tau}=\widetilde{v} \\
& \frac{d \widetilde{v}}{d \tau}=\left(\widetilde{u}-\bar{t}+\frac{1}{2}\right)\left(\tilde{u}^{2}-\frac{9}{4}\right), \\
& \frac{d \widetilde{w}}{d \tau}=\frac{3}{\sqrt{2}}(\tilde{u}-\widetilde{w})
\end{aligned}
$$

The corresponding characteristic equation is given by

$$
(\lambda+1)\left[\lambda^{2}-F_{y_{1}}\left(M_{1,2}\right)\right]=0 .
$$

By (45), we can solve the following characteristic roots:

$$
\begin{aligned}
\lambda_{1,2} & = \pm \sqrt{F_{y_{1}\left(M_{1,2}\right)}} \\
\lambda_{3} & =-1
\end{aligned}
$$

where $F_{y_{1}}\left(M_{1}\right)=3 \bar{t}+3>0$ and $F_{y_{1}}\left(M_{2}\right)=3(2-$ $\bar{t})>0$. So the two equilibrium points $M_{1}(-3 / 2,0,-3 / 2)$ and $M_{2}(3 / 2,0,3 / 2)$ are all hyperbolic saddle points of (43). To determine $t_{0}$, we can discuss the equations $Q_{0}^{( \pm)} \mathbf{y}(\tau)$ as follows:

$$
\begin{aligned}
& \frac{d Q_{0}^{(-)} u}{d \tau}=Q_{0}^{(-)} v \\
& \frac{d Q_{0}^{(-)} v}{d \tau} \\
& \quad=\left(-\frac{3}{2}+Q_{0}^{(-)} u-t_{0}+\frac{1}{2}\right)\left[\left(-\frac{3}{2}+Q_{0}^{(-)} u\right)^{2}-\frac{9}{4}\right], \\
& \frac{d Q_{0}^{(-)} w}{d \tau}=\frac{3}{\sqrt{2}}\left(Q_{0}^{(-)} u-Q_{0}^{(-)} w\right),
\end{aligned}
$$




$$
\begin{aligned}
& -\frac{3}{2}+Q_{0}^{(-)} u=t_{0}-\frac{1}{2} \\
& Q_{0}^{(-)} \mathbf{y}(-\infty)=0 \\
& \frac{d Q_{0}^{(+)} u}{d \tau}=Q_{0}^{(+)} v \\
& \frac{d Q_{0}^{(+)} v}{d \tau} \\
& \quad=\left(\frac{3}{2}+Q_{0}^{(+)} u-t_{0}+\frac{1}{2}\right)\left[\left(\frac{3}{2}+Q_{0}^{(+)} u\right)^{2}-\frac{9}{4}\right] \\
& \frac{d Q_{0}^{(+)} w}{d \tau}=\frac{3}{\sqrt{2}}\left(Q_{0}^{(+)} u-Q_{0}^{(+)} w\right), \\
& \frac{3}{2}+Q_{0}^{(+)} u=t_{0}-\frac{1}{2}, \\
& Q_{0}^{(+)} \mathbf{y}(+\infty)=0 .
\end{aligned}
$$

Assuming that $\widetilde{u}^{( \pm)}= \pm 3 / 2+Q_{0}^{( \pm)} u, \widetilde{v}^{( \pm)}=Q_{0}^{( \pm)} v$, and $\widetilde{w}^{( \pm)}=$ $\pm 3 / 2+Q_{0}^{( \pm)} w,(47)$ and (48) can be expressed by

$$
\begin{aligned}
\frac{d \widetilde{u}^{( \pm)}}{d \tau} & =\widetilde{v}^{( \pm)}, \\
\frac{d \widetilde{v}^{( \pm)}}{d \tau} & =\left(\widetilde{u}^{( \pm)}-t_{0}+\frac{1}{2}\right)\left[\left(\widetilde{u}^{( \pm)}\right)^{2}-\frac{9}{4}\right], \\
\frac{d \widetilde{w}^{( \pm)}}{d \tau} & =\frac{3}{\sqrt{2}}\left(\widetilde{u}^{( \pm)}-\widetilde{w}^{( \pm)}\right), \\
\widetilde{u}^{( \pm)}(0) & =t_{0}-\frac{1}{2} .
\end{aligned}
$$

The first integral of (49) passing through $M_{1}$ and $M_{2}$ is

$$
\begin{aligned}
{\left[\widetilde{v}^{( \pm)}(\tau)\right]^{2} } & =2 \int_{-(3 / 2)(3 / 2)}^{\widetilde{u}^{(\mp)}}\left(s-t_{0}+\frac{1}{2}\right)\left(s^{2}-\frac{9}{4}\right) d s \\
& =0 .
\end{aligned}
$$

Since the solutions of (41) are connected smoothly at the point of $t_{0}$, we have $\widetilde{u}^{(-)}(0)=\widetilde{u}^{(+)}(0)$. Assuming that

$$
H\left(t_{0}\right)=\widetilde{u}^{(-)}(0)-\widetilde{u}^{(+)}(0)=0,
$$

substituting (50) into (51), we can obtain

$$
\int_{-3 / 2}^{3 / 2}\left(s-t_{0}+\frac{1}{2}\right)\left(s^{2}-\frac{9}{4}\right) d s=0
$$

On the basis of (52), we can solve $t_{0}=1 / 2$ and $\partial H\left(t_{0}\right) / \partial t_{0}=$ $21 / 4 \neq 0$. So the solutions of (41) transfer at the point of $t_{0}=$ $1 / 2$.
Substituting $t_{0}=1 / 2$ into (49), as $\tau \leq 0$, we have

$$
\begin{aligned}
& \widetilde{u}^{(-)}(\tau)=\frac{3 C e^{(3 / \sqrt{2}) \tau}+3}{2 C e^{(3 / \sqrt{2}) \tau}-2}, \\
& \widetilde{v}^{(-)}(\tau)=\frac{9 \sqrt{2} C e^{-3 / \sqrt{2}} \tau}{4\left(1-C e^{(3 / \sqrt{2}) \tau}\right)^{2}}, \\
& \widetilde{w}^{(-)}(\tau)=\frac{3}{2}+\frac{2 \ln \left|C e^{(3 / \sqrt{2}) \tau}-1\right|}{C e^{(3 / \sqrt{2}) \tau}} .
\end{aligned}
$$

As $\tau \geq 0$, we can solve

$$
\begin{aligned}
& \tilde{u}^{(+)}(\tau)=\frac{3 C+3 e^{-(3 / \sqrt{2}) \tau}}{2 C-2 e^{-(3 / \sqrt{2}) \tau}}, \\
& \widetilde{v}^{(+)}(\tau)=\frac{9 \sqrt{2} C e^{-3 / \sqrt{2}} \tau}{4\left(1-C e^{-(3 / \sqrt{2}) \tau}\right)^{2}}, \\
& \widetilde{w}^{(+)}(\tau)=\frac{3}{2}+\frac{2 \ln \left|C e^{-(3 / \sqrt{2}) \tau}-1\right|}{C e^{-(3 / \sqrt{2}) \tau}} .
\end{aligned}
$$

So we can solve the interior-layer functions as follows:

$$
\begin{aligned}
& Q_{0}^{(-)} u(\tau)=\frac{3 C e^{(3 / \sqrt{2}) \tau}}{C e^{(3 / \sqrt{2}) \tau}-1}, \\
& Q_{0}^{(-)} v(\tau)=\frac{9 \sqrt{2} C e^{-3 / \sqrt{2}} \tau}{4\left(1-C e^{(3 / \sqrt{2}) \tau}\right)^{2}}, \\
& Q_{0}^{(-)} w(\tau)=3+\frac{2 \ln \left|C e^{(3 / \sqrt{2}) \tau}-1\right|}{C e^{(3 / \sqrt{2}) \tau}}, \\
& Q_{0}^{(+)} u(\tau)=\frac{3 e^{-(3 / \sqrt{2}) \tau}}{C-e^{-(3 / \sqrt{2}) \tau},} \\
& Q_{0}^{(+)} v(\tau)=\frac{9 \sqrt{2} C e^{-(3 / \sqrt{2})} \tau}{4\left(1-C e^{-(3 / \sqrt{2}) \tau}\right)^{2}}, \\
& Q_{0}^{(+)} w(\tau)=\frac{2 \ln \left|C e^{-(3 / \sqrt{2}) \tau}-1\right|}{C e^{-(3 / \sqrt{2}) \tau}} .
\end{aligned}
$$

Similarly, the boundary layer function $L_{0} \mathbf{y}\left(\tau_{0}\right)$ can be expressed by

$$
\begin{aligned}
& L_{0} u\left(\tau_{0}\right)=\frac{3 e^{-(3 / \sqrt{2}) \tau_{0}}}{e^{-(3 / \sqrt{2}) \tau_{0}-A},} \\
& L_{0} v\left(\tau_{0}\right)=\frac{9 \sqrt{2} C e^{-(3 / \sqrt{2})} \tau_{0}}{4\left(1-A e^{-(3 / \sqrt{2}) \tau_{0}}\right)^{2}}, \\
& L_{0} w\left(\tau_{0}\right)=\frac{2 \ln \left|A e^{-(3 / \sqrt{2}) \tau_{0}}-1\right|}{A e^{-(3 / \sqrt{2}) \tau_{0}}} .
\end{aligned}
$$


The boundary layer function $R_{0} \mathbf{y}\left(\tau_{1}\right)$ can be expressed by

$$
\begin{aligned}
& R_{0} u\left(\tau_{1}\right)=\frac{3 e^{(3 / \sqrt{2}) \tau_{1}}}{B-e^{(3 / \sqrt{2}) \tau_{1}}} \\
& R_{0} v\left(\tau_{1}\right)=\frac{9 \sqrt{2} C e^{3 / \sqrt{2}} \tau_{1}}{4\left(1-B e_{1}^{(3 / \sqrt{2}) \tau}\right)^{2}}, \\
& R_{0} w\left(\tau_{1}\right)=-3+\frac{2 \ln \left|A e^{(3 / \sqrt{2}) \tau_{1}}-1\right|}{A e^{(3 / \sqrt{2}) \tau_{1}}},
\end{aligned}
$$

so we can construct a zero-order asymptotic solution of (41) and (42) as follows:

$$
\begin{aligned}
& \mathbf{x}(t, \mu) \\
& = \begin{cases}\overline{\mathbf{x}}_{0}^{(-)}(t)+L_{0} \mathbf{x}(\tau)+Q_{0}^{(-)} \mathbf{x}(\tau)+O(\mu), & 0 \leq t \leq t^{*}, \\
\overline{\mathbf{x}}_{0}^{(+)}(t)+Q_{0}^{(+)} \mathbf{x}(\tau)+R_{0} \mathbf{x}\left(\tau_{1}\right)+O(\mu), & t^{*} \leq t \leq 1,\end{cases}
\end{aligned}
$$

where $t^{*}=1 / 2+O(\mu), \overline{\mathbf{x}}_{0}^{(-)}(t)=(-3 / 2,0,-3 / 2)^{T}$, and $\overline{\mathbf{x}}_{0}^{(+)}(t)=(3 / 2,0,3 / 2)^{T}$.

\section{Conclusive Remarks}

By the boundary layer function method and smooth connection, we study the contrast structure for a class of semilinear singularly perturbed systems. Under some assumptions, the existence of a step-like contrast structure of system (3) and a heteroclinic orbit connecting two equilibrium points of the corresponding associated systems is determined. Then, we obtain the asymptotic solution of system (3). In comparison with $[8,9]$, the system we study is more general.

\section{Conflict of Interests}

The authors declare that there is no conflict of interests regarding the publication of this paper.

\section{Authors' Contribution}

Han Xu completed the main part of this paper, and Yinlai Jin corrected the main theorems.

\section{Acknowledgments}

This work is supported by the National Natural Science Funds (no. 11201211), Shandong Province Higher Educational Science and Technology Program (no. J13LI56), Shandong Province Higher Educational Youth Backbone Teachers Domestic Visiting Scholars Project, and Applied Mathematics Enhancement Program of Linyi University.

\section{References}

[1] V. F. Butuzov, A. B. Vasileva, and N. N. Nefedov, "The asymptotic theory of contrast structures," Automation and Remote Control, vol. 3, pp. 4-32, 1997.
[2] A. B. Vasileva, V. F. Butuzov, and N. N. Nefedov, "Contrast structures in singularly perturbed problems," Fundamentalnaya i Prikladnaya Matematika, vol. 4, no. 3, pp. 799-851, 1998.

[3] A. F. Wang, "The spike-type contrast structure for a secondorder semi-linear singularly perturbed differential equation with integral boundary condition," Mathematica Applicata, vol. 25, no. 2, pp. 363-368, 2012.

[4] X.-B. Lin, "Heteroclinic bifurcation and singularly perturbed boundary value problems," Journal of Differential Equations, vol. 84, no. 2, pp. 319-382, 1990.

[5] Y. L. Jin, F. Li, H. Xu, J. Li, L. Zhang, and B. Ding, "Bifurcations and stability of nondegenerated homoclinic loops for higher dimensional systems," Computational and Mathematical Methods in Medicine, vol. 2013, Article ID 582820, 9 pages, 2013.

[6] Y. L. Jin, X. W. Zhu, Z. Guo, H. Xu, L. Zhang, and B. Ding, "Bifurcations of nontwisted heteroclinic loop with resonant eigenvalues," The Scientific World Journal, vol. 2014, Article ID 716082, 8 pages, 2014.

[7] M. K. Ni and Z. M. Wang, "On higher-dimensional contrast structure of singularly perturbed Dirichlet problem," Science China Mathematics, vol. 55, no. 3, pp. 495-507, 2012.

[8] A. F. Wang, "The step-type contrast structure for a singularly perturbed system with slow and fast variables," Journal of Shandong University, vol. 48, no. 2, pp. 98-104, 2013.

[9] Z. N. Ma and Y. C. Zhou, The Mathematics Modeling about Epidemic Dynamics, Science Press, Beijing, China, 2004.

[10] J. Chattoadhyay and N. Bairagi, "Pelicans at risk in salton sea an ecoepidemiological model," Ecological Modelling, vol. 136, no. 2-3, pp. 103-112, 2001.

[11] $\mathrm{H} . \mathrm{Xu}$, "The two-order quasi-linear singular perturbed problems with infinite initial conditions," Advances in Civil and Industrial Engineering, vol. 1, pp. 3248-3250, 2013.

[12] H. Xu and Y. L. Jin, "The asymptotic solutions for a class of nonlinear singular perturbed differential systems with time delays," The Scientific World Journal, vol. 2014, Article ID 965376, 7 pages, 2014. 


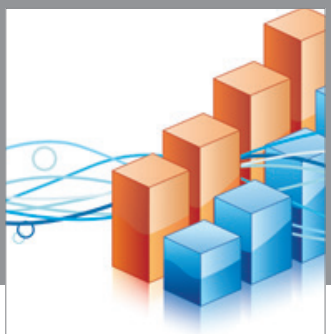

Advances in

Operations Research

vatem alat4

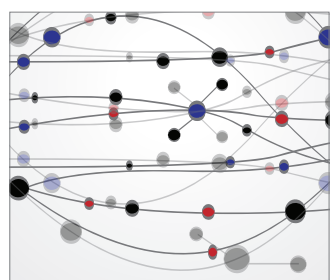

\section{The Scientific} World Journal
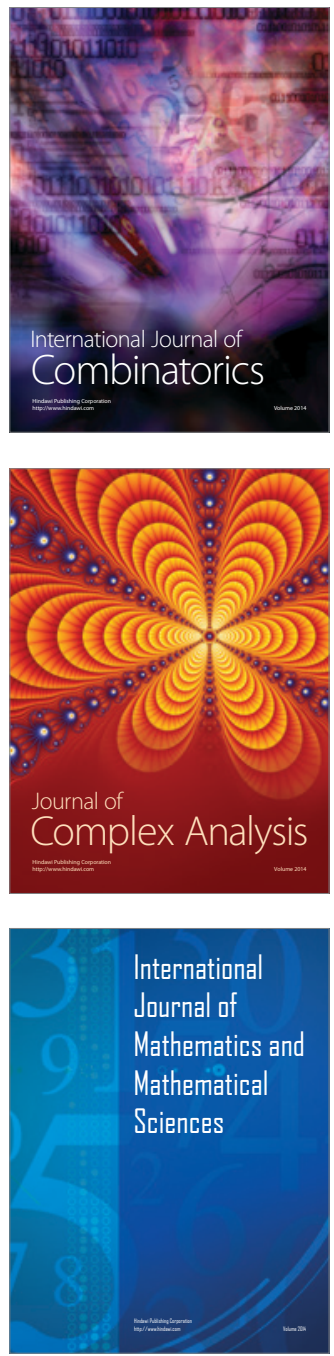
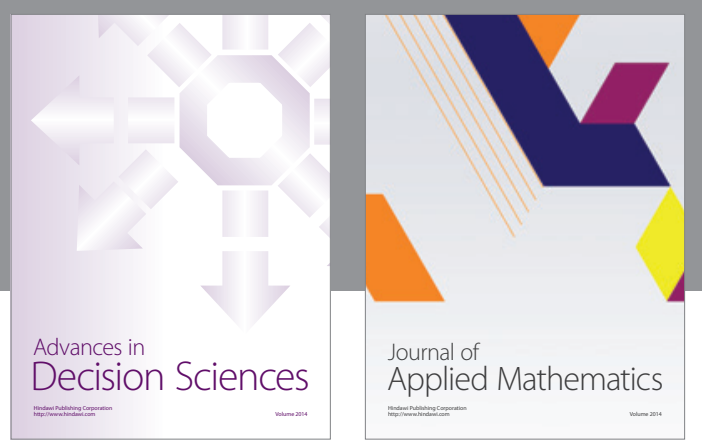

Algebra

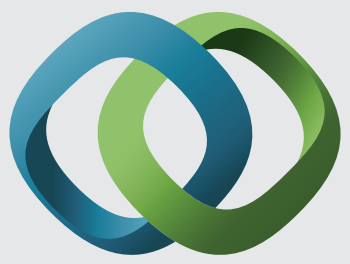

\section{Hindawi}

Submit your manuscripts at

http://www.hindawi.com
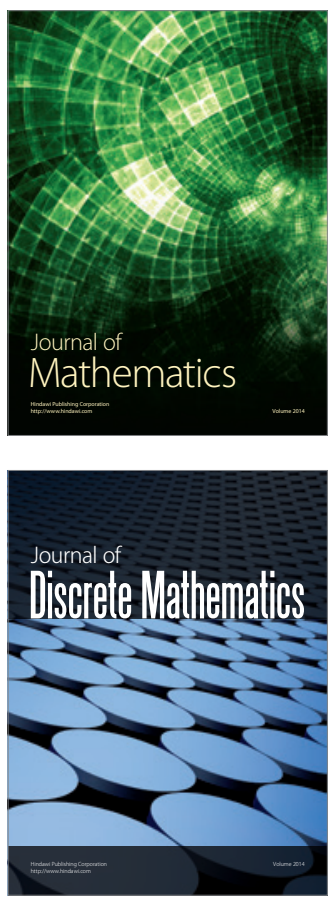

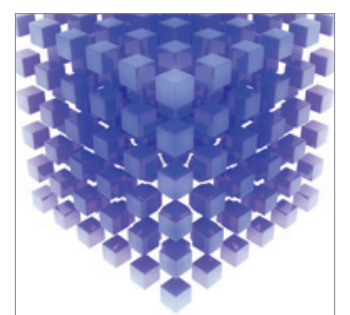

Mathematical Problems in Engineering
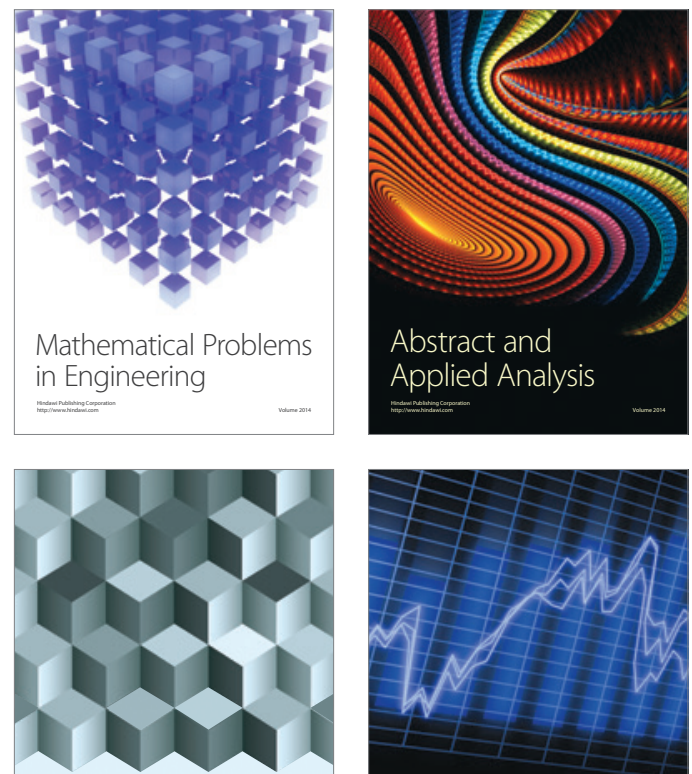

Journal of

Function Spaces

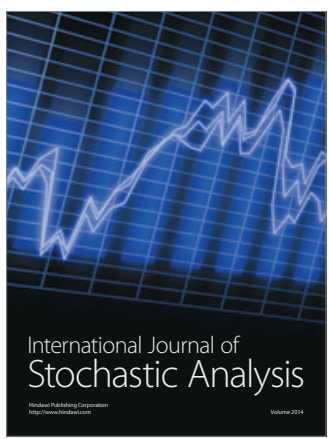

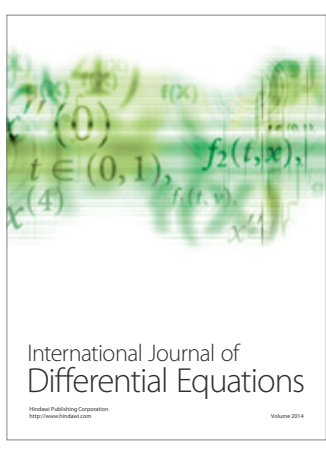
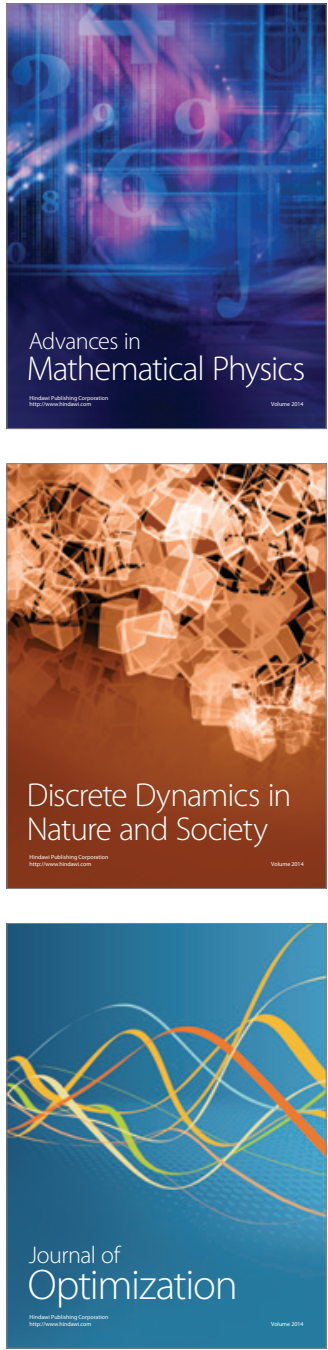